\title{
Optical bandgap of semiconductor nanostructures: Methods for experimental data analysis
}

R. Raciti, R. Bahariqushchi, C. Summonte, A. Aydinli, A. Terrasi, and S. Mirabella

Citation: Journal of Applied Physics 121, 234304 (2017); doi: 10.1063/1.4986436

View online: https://doi.org/10.1063/1.4986436

View Table of Contents: http://aip.scitation.org/toc/jap/121/23

Published by the American Institute of Physics

\section{Articles you may be interested in}

Shell morphology and Raman spectra of epitaxial $\mathrm{Ge}_{-} \mathrm{Si}_{x} \mathrm{Ge}_{1-x}$ and $\mathrm{Si}_{-} \mathrm{Si}_{x} \mathrm{Ge}_{1-x}$ core-shell nanowires

Journal of Applied Physics 121, 234302 (2017); 10.1063/1.4985616

Crystal and band structures of ZnS, MgS, and ZnS-MgS alloys

Journal of Applied Physics 121, 235705 (2017); 10.1063/1.4986929

Defect analysis in GaN films of HEMT structure by cross-sectional cathodoluminescence

Journal of Applied Physics 121, 235703 (2017); 10.1063/1.4986497

Exciton center-of-mass localization and dielectric environment effect in monolayer WS2

Journal of Applied Physics 121, 235702 (2017); 10.1063/1.4984790

The effect of an InP cap layer on the photoluminescence of an $\ln _{x} \mathrm{Ga}_{1-x} \mathrm{As}_{1-y} \mathrm{P} \mathrm{P}_{\mathrm{y}} / \mathrm{In}_{z} \mathrm{Al}_{1-z} \mathrm{As}$ quantum well heterostructure

Journal of Applied Physics 121, 235301 (2017); 10.1063/1.4985614

All-optical measurements of carrier dynamics in bulk-GaN LEDs: Beyond the ABC approximation

Applied Physics Letters 110, 253504 (2017); 10.1063/1.4986908

\section{PHYSICS TODAY}

MANAGER'S GUIDE

Accelerate R\&D with

Multiphysics Simulation

\section{READ NOW}

PRESENTED BY

○О $\subset$ MSOL 


\title{
Optical bandgap of semiconductor nanostructures: Methods for experimental data analysis
}

\author{
R. Raciti, ${ }^{1}$ R. Bahariqushchi, ${ }^{2}$ C. Summonte,${ }^{3}$ A. Aydinli, ${ }^{2,4}$ A. Terrasi, ${ }^{1}$ and S. Mirabella ${ }^{1}$ \\ ${ }^{1}$ MATIS CNR-IMM and Dipartimento di Fisica e Astronomia, Università di Catania, via S. Sofia 64, \\ 95123 Catania, Italy \\ ${ }^{2}$ Department of Physics, Bilkent University, 06800 Ankara, Turkey \\ ${ }^{3}$ IMM-CNR, via Gobetti, 101-40129 Bologna, Italy \\ ${ }^{4}$ Department of Electrical and Electronics Engineering, Uludag University, 16059 Bursa, Turkey
}

(Received 10 February 2017; accepted 3 June 2017; published online 20 June 2017)

\begin{abstract}
Determination of the optical bandgap $\left(E_{g}\right)$ in semiconductor nanostructures is a key issue in understanding the extent of quantum confinement effects (QCE) on electronic properties and it usually involves some analytical approximation in experimental data reduction and modeling of the light absorption processes. Here, we compare some of the analytical procedures frequently used to evaluate the optical bandgap from reflectance $(\mathrm{R})$ and transmittance $(\mathrm{T})$ spectra. Ge quantum wells and quantum dots embedded in $\mathrm{SiO}_{2}$ were produced by plasma enhanced chemical vapor deposition, and light absorption was characterized by UV-Vis/NIR spectrophotometry. R\&T elaboration to extract the absorption spectra was conducted by two approximated methods (single or double pass approximation, single pass analysis, and double pass analysis, respectively) followed by $\mathrm{E}_{\mathrm{g}}$ evaluation through linear fit of Tauc or Cody plots. Direct fitting of R\&T spectra through a TaucLorentz oscillator model is used as comparison. Methods and data are discussed also in terms of the light absorption process in the presence of QCE. The reported data show that, despite the approximation, the DPA approach joined with Tauc plot gives reliable results, with clear advantages in terms of computational efforts and understanding of QCE. Published by AIP Publishing.
\end{abstract}

[http://dx.doi.org/10.1063/1.4986436]

\section{INTRODUCTION}

In the past decade, several studies focused on the optical properties of semiconductor nanostructures (NS). As the size of a semiconductor material is reduced below exciton Bohr radius ( $5 \mathrm{~nm}$ for $\mathrm{Si}$ and $24 \mathrm{~nm}$ for $\mathrm{Ge}^{1,2}$ ), the appearance of interesting optical features is expected due to quantum confinement effects (QCEs). Two of the most important optical properties of NS in the quantum confinement regime are the increase in the optical bandgap $\left(\mathrm{E}_{\mathrm{g}}\right)$ and oscillator strength with the size reduction. ${ }^{3}$ These characteristics allow tailoring of the light absorption and emission spectra, making possible the application of NS in many fields such as photovoltaics, ${ }^{4}$ photodetection, ${ }^{5-7}$ and optoelectronic ${ }^{8,9}$ applications. Actually, the absorption modulation and enhancement in NS are still under investigation, since other factors ${ }^{10-16}$ can interfere with the QCE. In fact, several studies have recently demonstrated how the optical properties of $\mathrm{Si}$ and Ge NS can be changed by varying several structural characteristics such as shape, ${ }^{10}$ crystalline structure, ${ }^{11,12}$ or the potential barriers surrounding the NS. ${ }^{13-16}$

For all these reasons, an accurate extraction of optical parameters from experimental data becomes crucial, in order to have a deeper and quantitative understanding of QCE. To date, a long debate has been underway on how to extract the optical bandgap from experimental data. Among different experimental techniques, UV-Vis/NIR spectrophotometry is the most widely used to measure transmittance (T) and reflectance (R) spectra. Based on R\&T spectra, two steps are commonly used: (i) the absorption coefficient $\alpha$ is extracted; (ii) the optical bandgap $\mathrm{E}_{\mathrm{g}}$ is determined by means of linear extrapolation with Tauc or Cody plots. ${ }^{17,18}$ Concerning the first step, an approximate analysis exists labeled as the single pass analysis (SPA), where the incident light propagates through the film, neglecting multiple reflections at the film/ substrate interface and interference. In this case, the absorption coefficient $(\alpha)$ following the Beer-Lambert law can be simply extracted by using the following equation:

$$
\alpha=\frac{1}{d} \ln \frac{\left(1-R_{S}\right)}{T_{s}},
$$

where $\mathrm{d}, \mathrm{T}_{\mathrm{s}}$, and $\mathrm{R}_{\mathrm{s}}$ are thickness, transmittance, and reflectance of sample, respectively. Once the absorption coefficient spectra are extracted, the Tauc model or Cody model are commonly applied in order to extract the optical bandgap. The Tauc model is based on the constant momentum matrix approximation, so that the energy dependence of $\alpha$ in amorphous semiconductors is satisfactorily modelled by the following equation:

$$
\alpha=\frac{B}{h \nu}\left(h \nu-E_{g}\right)^{2}
$$

where $\mathrm{h} \nu$ is the energy of incoming photons, and $\mathrm{B}$ is the Tauc coefficient describing the efficiency in light absorption. ${ }^{17}$ Clearly, the optical parameters $\mathrm{E}_{\mathrm{g}}$ and $\mathrm{B}$ can be extracted through linear fit of $\sqrt{\alpha h \nu}$ (Tauc plot). The Cody model is based on a constant dipole matrix approximation 
and a different expression for the absorption coefficient is used

$$
\alpha \propto h \nu\left(h \nu-E_{g}\right)^{2} .
$$

Now $E_{\mathrm{g}}$ is extracted through a linear fit of $\sqrt{\frac{\alpha}{h \nu}}$ (Cody plot). ${ }^{18}$ On the choice among Tauc or Cody plots, many papers are present in the literature. ${ }^{17-21}$ Most of them focus on the light absorption in a-Si and a-Si:H, debating on the effect and magnitude of tail states in the band gap and on the validity of $\mathrm{Tauc}^{20}$ or Cody ${ }^{21}$ model. Recently, the two models have been compared in sputtered Ge quantum well (QWs), ${ }^{19}$ showing the presence of a double slope in the Tauc plot and claiming that the Cody plot is able to provide a more unambiguous determination of the optical bandgap compared to Tauc plot. ${ }^{19}$ Actually, since the models use different approximations and the linear fit to extract $\mathrm{E}_{\mathrm{g}}$ have been done in a large variety of energy ranges (from 0.3 to $2 \mathrm{eV}$ ), special care must be taken to compare the literature results. The choice itself of using the approximated expression (1) may impact on the results. In fact, neglecting reflections at the interfaces causes an increasing inaccuracy for thinner films, highly absorbing materials, and/or materials that show large difference in the refractive index with respect to the substrate.

In this work, we compare three analytical methods to extract $\mathrm{E}_{\mathrm{g}}$ from $\mathrm{R} \& \mathrm{~T}$ experimental data, with the final aim to show pros and cons in terms of complexity and accuracy. We used Ge QWs as they join the simplest confining structure $(\mathrm{QW})$ and a semiconductor material $(\mathrm{Ge})$ with a relative large Bohr radius QCE. The Ge QWs were produced by plasma enhanced chemical vapor deposition (PECVD), as they exhibit a much cleaner and sharper interface compared to Ge NS produced by sputtering techniques, allowing a stronger quantum confinement effect. ${ }^{16}$ Moreover, the use of a high refractive index material, such as Ge, in films a few nanometers thin, is a situation that enhances the impact of the approximations, thus representing the most stringent test bed for the optical models. Both the extractions of $\alpha$ from $\mathrm{R} \& \mathrm{~T}$ spectra and the determination of $\mathrm{E}_{\mathrm{g}}$ from $\alpha$ are discussed. A simple method (labeled as double pass DPA) is then presented, showing that it is able to determine $\mathrm{E}_{\mathrm{g}}$ with the same accuracy as the non-approximated method. The methods were compared also for Ge quantum dots (QDs) embedded in $\mathrm{SiO}_{2}$, to show the validity in other confined structures. The results are linked to the effect of quantum confinement in semiconductor nanostructures, allowing the application of the methodology to a large variety of semiconductors nanostructures.

\section{METHODS}

For $\mathrm{Ge}$ QWs, a $\mathrm{SiO}_{2}(20 \mathrm{~nm}) / \mathrm{Ge} / \mathrm{SiO}_{2}(20 \mathrm{~nm})$ structure was deposited on fused silica quartz at $250{ }^{\circ} \mathrm{C}$ by plasma enhanced chemical vapor deposition (PECVD). Different thicknesses $(4,6,8 \mathrm{~nm})$ of Ge film were obtained by varying the time of deposition and keeping constant the flux of $\mathrm{GeH}_{4}$. One reference Ge film, $120 \mathrm{~nm}$ thick, was also grown without the presence of a $\mathrm{SiO}_{2}$ buffer layer. The atomic Ge content and the thickness of the films were evaluated by Rutherford backscattering spectrometry (RBS), using a $2.0 \mathrm{MeV} \mathrm{He}^{+}$beam in glancing detection configuration (backscattering angle of $105^{\circ}$ ) to enhance the depth resolution, and employing SIMNRA software. ${ }^{22}$ Transmittance and reflectance spectra were acquired using a Varian Cary 500 double beam scanning UV/visible/NIR spectrophotometer, as described in Refs. 11 and 23. For Ge QDs, thin films containing Si:Ge:O alloy were deposited by PECVD $(\sim 300 \mathrm{~nm}$ thick) on fused silica quartz, followed by thermal annealing at $800^{\circ} \mathrm{C}$ for $1 \mathrm{~h}$ to induce the precipitation of the excess of Ge in QDs. Raman spectroscopy performed on annealed samples at $800^{\circ} \mathrm{C}$ revealed the presence of a considerable fraction of amorphous Ge QDs while Transmission Electron Microscopy (TEM) analysis estimated a QDs mean size of $8 \mathrm{~nm} .{ }^{23}$ TEM electron energy loss spectroscopy (EELS) analysis also excluded the presence of other aggregates as $\mathrm{Si}$ and SiGe QDs. ${ }^{16}$ Here, we briefly review the three models used for the extraction of optical bandgap in our samples [Fig. 1(a)].

The accurate model, known as Jellison Tauc Lorentz model (labelled as JTL model in the following), is based on

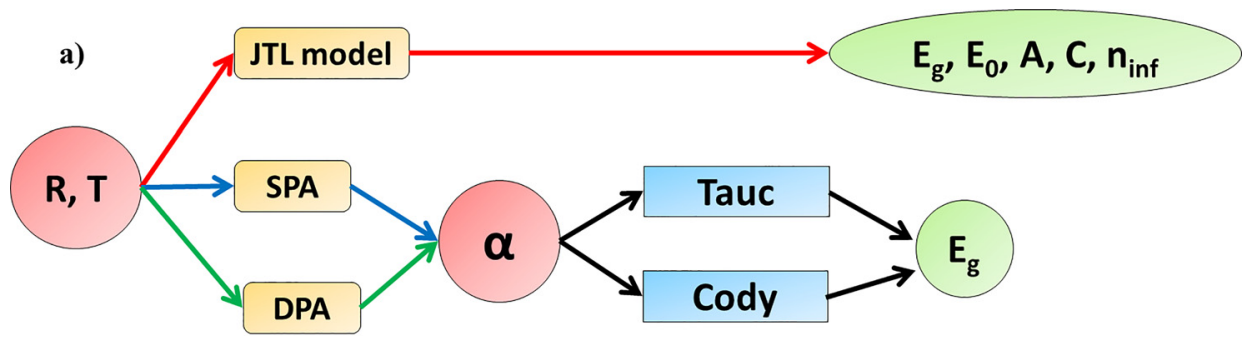

FIG. 1. (a) Schematic picture of methods for extraction of optical bandgap $\mathrm{E}_{\mathrm{g}}$ : JTL method (red path) and approximated methods [DPA (violet path) and SPA (blue path)]. Drawing of light

b) JTL model

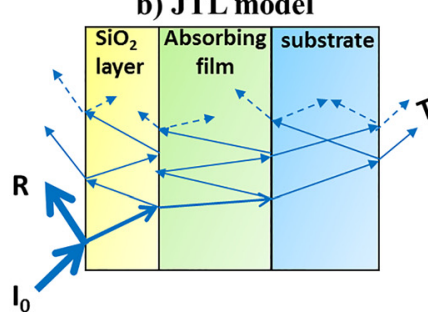

c) DPA model

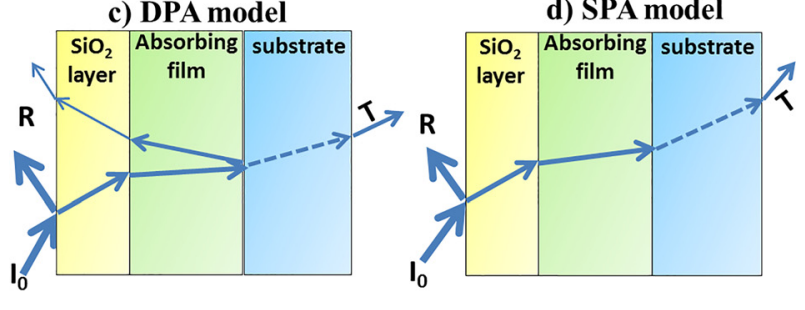

paths in JTL (b), DPA (c), and SPA (d) models. 
the simulation of the R\&T spectra by means of the Generalized Transfer Method (GTM), ${ }^{24}$ which takes into account the reflection and transmission at all interfaces, and makes use of the complex spectral refractive index of the involved materials (film and substrate). The complex spectral refractive index of the unknown film is modeled by means of the Tauc-Lorentz approximation, in the form proposed by Jellison and Modine in $1996 .^{25}$ In this method, the imaginary part of the dielectric function $\left(\varepsilon_{2}\right)$ is determined by combining a single classical Lorentz oscillator with the absorption decay deriving from the Tauc joint density of states. The TaucLorentz approximation can be considered an accurate way of modelling light absorption in amorphous semiconductor thin films. On the basis of this model, the imaginary part of dielectric function $\varepsilon_{2}$ is given by the following expression: ${ }^{25-27}$

$$
\begin{cases}\varepsilon_{2}=\frac{1}{E} \frac{A E_{0} C\left(E-E_{g}\right)^{2}}{\left(E^{2}-E_{0}^{2}\right)^{2}+C^{2} E^{2}} & \text { for } E>E_{g} \\ \varepsilon_{2}=0 & \text { for } E<E_{g},\end{cases}
$$

where $E_{g}$ is the band-gap of the material, $A$ is the oscillator amplitude, $\mathrm{E}_{0}$ is the energy position of the Lorentz peak, and $\mathrm{C}$ is the broadening parameter. The real part $\varepsilon_{1}$ of dielectric function is derived from the expression of $\varepsilon_{2}$ using the Kramers and Kronig integration, as follows: ${ }^{25-27}$

$$
\varepsilon_{1}=\varepsilon_{1}(\infty)+\frac{2}{\pi} \cdot P \cdot \int_{E_{g}}^{\infty} \frac{\xi \cdot \varepsilon_{2}(\xi)}{\xi^{2}-E^{2}} d \xi,
$$

where the $\mathrm{P}$ stands for the Cauchy principal part of the integral and an additional fitting parameter $\varepsilon_{1}(\infty)$ has been included. The fit parameters of this model are five $\left[\mathrm{E}_{\mathrm{g}}, \mathrm{E}_{0}, \mathrm{~A}\right.$, $\mathrm{C}$, and $\varepsilon_{1}(\infty)$ ] leading to the $(\mathrm{n}, \mathrm{k})$ spectra which are used, through the general transfer method, to simulate R\&T, which are then compared to the experimental ones. Iterative fitting cycles based on $\chi^{2}$ minimization are then used to determine the set of parameters that supply the best fit between simulated and experimental R\&T spectra. The fitting was performed using the GTB-fit computer programme, ${ }^{26}$ which is based on the Optical code. ${ }^{24}$ Both programmes are open source and available online. Further details can be found in Refs. 24-26. A sketch of the procedure is illustrated in Fig. 1(b).

The SPA and DPA, shown in Fig. 1, are approximated methods, as the absorption coefficient spectrum is directly extracted from experimental R\&T data, by partially or totally neglecting the multiple reflections and interference effects. In the SPA model [blue arrows in Fig. 1(a)] even the first reflection at film/substrate interface is neglected, as reported in Fig. 1(d). So, the absorption coefficient can be extracted by using Eq. (1). In DPA model [green arrows in Fig. 1(a)], the first reflection between absorbing thin film and substrate, if any, is taken in account, as reported in Fig. 1(c). In this way, the absorption coefficient spectra can be expressed as follows:

$$
\alpha=\frac{1}{d} \ln \frac{T_{s u b}\left(1-R_{S}\right)}{T_{s}},
$$

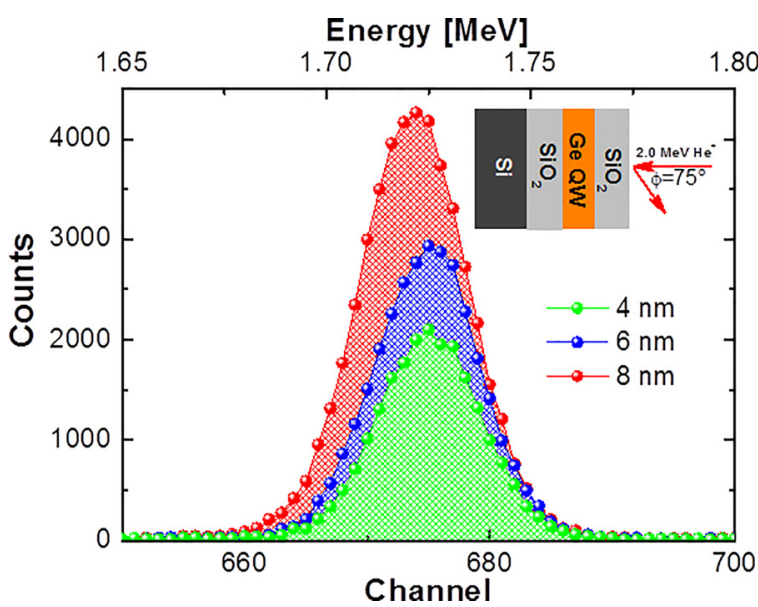

FIG. 2. RBS spectra of a-Ge QWs. The inset image represents the schematic of experimental setup.

where $\mathrm{T}_{\text {sub }}$ is the substrate transmittance. ${ }^{11}$ Once the absorption coefficient spectra is calculated through Eq. (1) or (6), Tauc or Cody plots are applied to extract optical bandgaps through linear fit. In summary, in the above three methods, $\mathrm{E}_{\mathrm{g}}$ is extracted in different ways. In JTL method, the Tauc gap $E_{g}$ is a fitting parameter of the R\&T spectra. In the other methods, $\mathrm{E}_{\mathrm{g}}$ is obtained by linear fitting (Tauc or Cody plots) of $\alpha$ extracted directly from R\&T data (DPA or SPA methods) or calculated by (n-k) dispersion derived by fitting R\&T (GTM methods). In the following, JTL, DPA, and SPA methods will be compared on experimental data of Ge QWs and QDs.

\section{EXPERIMENTAL DATA}

Figure 2 reports the RBS data in the $1.68-1.78 \mathrm{MeV}$ energy range, which are relative to $\mathrm{He}^{+}$backscattered by Ge atoms. The peak area is proportional to the Ge atomic dose contained in each QW. The extracted Ge dose of our samples varied from $1.7 \times 10^{16} \mathrm{at} / \mathrm{cm}^{2}$ for the thinnest film to $3.6 \times 10^{16} \mathrm{at} / \mathrm{cm}^{2}$ for the thickest one. By assuming the density for monocrystalline $\mathrm{Ge}\left(4.4 \times 10^{22} \mathrm{at} / \mathrm{cm}^{3}\right)$ for the deposited material, the thickness of each sample was estimated as

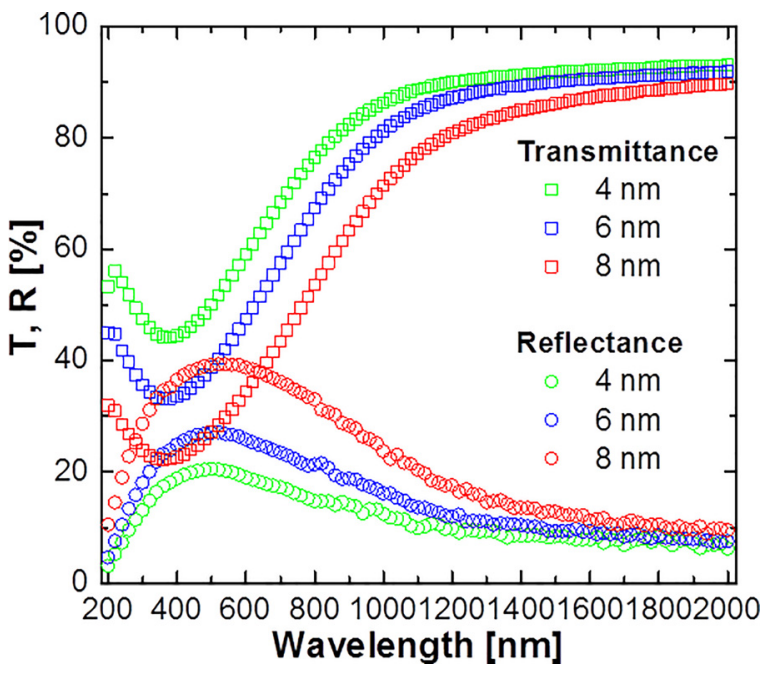

FIG. 3. Experimental T and R spectra of 4, 6, and $8 \mathrm{~nm}$ Ge QW. 

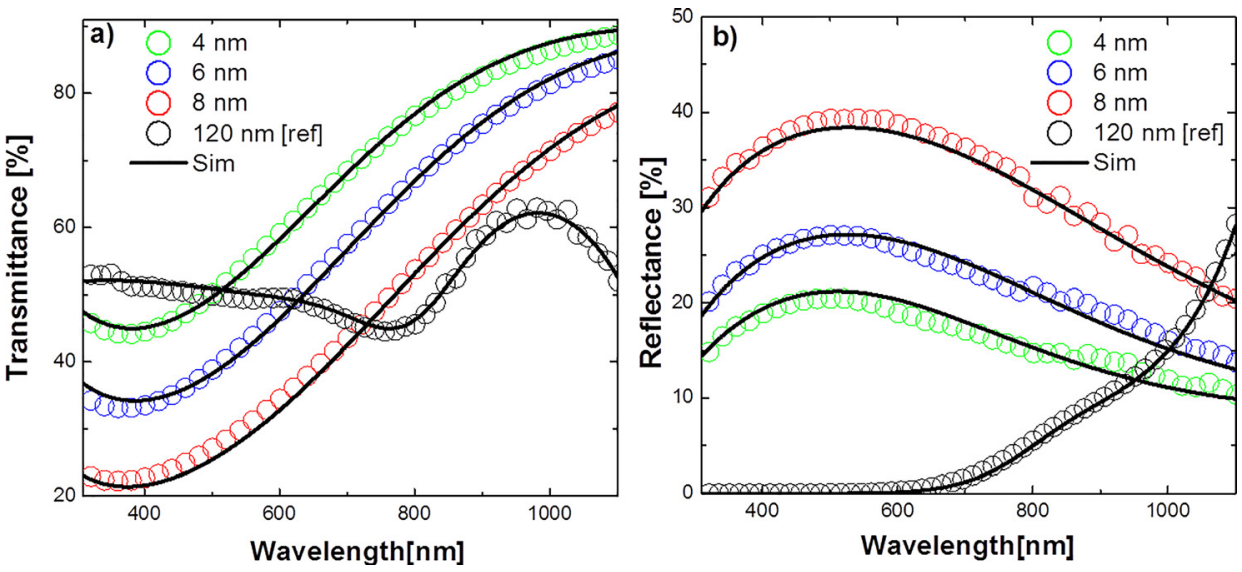

FIG. 4. Experimental (symbols) and computed (black lines) $\mathrm{T}$ (a) and $\mathrm{R}(\mathrm{b})$ spectra of a-Ge QW with different thickness and of a reference sample $(120 \mathrm{~nm}$ Ge film).
TABLE I. The five JTL fit parameters and the $\mathrm{X}^{2}$ test of all samples.

\begin{tabular}{lcccccc}
\hline \hline Sample $(\mathrm{nm})$ & $\mathrm{E}_{\mathrm{g}}(\mathrm{eV})$ & $\mathrm{E}_{0}(\mathrm{eV})$ & $\mathrm{A}$ & $\mathrm{C}(\mathrm{eV})$ & $\mathrm{n}_{\text {inf }}$ & $\chi^{2}$ \\
\hline 4 & 1.14 & 2.8 & 206 & 4.10 & 1.3 & 0.89 \\
6 & 1.05 & 2.8 & 201 & 3.96 & 1.3 & 0.59 \\
8 & 0.98 & 2.9 & 185 & 3.79 & 1.3 & 0.79 \\
120 & 0.88 & 2.6 & 175 & 3.34 & 1.3 & 0.32 \\
\hline \hline
\end{tabular}

the ratio between the Ge dose, measured by RBS, and the atomic density of Ge. Such an approach was verified by comparison with Transmission Electron Microscopy (TEM) measurements of film thickness in similar samples. ${ }^{28}$ The soobtained thicknesses of Ge QWs were 4, 6, and $8 \mathrm{~nm}$. The overall error on thickness, including the error on Ge dose, is about $5 \%$.

Figure 3 reports R\&T spectra of Ge films with different thicknesses. The increase in thickness produces a decrease of $\mathrm{T}$ in the UV-Vis region for all the investigated samples. While in the IR region $\mathrm{R}+\mathrm{T}=1$, in the $\mathrm{UV}-\mathrm{V}$ is region $\mathrm{T}+\mathrm{R}<1$ indicating that part of the incident light is absorbed by the Ge QW. In the following, experimental R\&T spectra will be analyzed through the three models [Fig. 1(b)], and a comparison will then be discussed.

\section{OPTICAL BANDGAP DETERMINATION}

\section{A. JTL approach}

To launch the inversion software GTB-fit, ${ }^{26}$ the structure " $\mathrm{SiO}_{2}(20 \mathrm{~nm}) /$ unknown film $/ \mathrm{SiO}_{2}$ substrate" was used.
The interfacial $\mathrm{SiO}_{2}$ layer is neglected, as it is optically indistinguishable from the substrate. For the unknown films, the thickness was fixed at the value determined as described in Sec. III. The optical constants of the $\mathrm{SiO}_{2}$ layer and substrate were taken from Ref. 29. In order to set the initial guess values for five JTL parameters, we start our analysis with a reference bulk Ge sample where QCE is truly absent, but the sample structure is maintained. In this case, the Ge film was $120 \mathrm{~nm}$ thick. A good match between the experimental and computed R\&T spectra is achieved, as reported in Fig. 4 (black symbols). The parameters providing the best fit are reported in the Table I. The optical bandgap $(0.88 \mathrm{eV})$ is in very good agreement with literature data $(0.8 \mathrm{eV}),{ }^{17-28}$ as well as the oscillator energy $(2.6 \mathrm{eV})$ which resembles the lower direct transition energy $\left(\mathrm{E}_{1}\right)$ of $\mathrm{c}-\mathrm{Ge}(2.5 \mathrm{eV}){ }^{30}$

For what concerns the Ge QWs simulations, the five JTL parameters have been set to initial values found in the reference Ge sample. Then, through iterative cycles, the best fit was obtained. For all samples, the set of best parameters is reported as table in Fig. 4. It should be noted that relative variation of $2 \%-3 \%$ in the values of $\mathrm{A}, \mathrm{E}_{\mathrm{g}}, \mathrm{E}_{0}$, and $\mathrm{C}$ does not significantly worsen the relative fit ensuring a well lower than 1.

As reported in Table $\mathrm{I}$, both $\mathrm{E}_{0}$ and $\mathrm{E}_{\mathrm{g}}$ increase with the reduction of the Ge QW thickness. These effects are due to QCE, in agreement with literature. ${ }^{31}$ Another significant effect due to confinement can be observed in our simulation parameters in terms of increase in the broadening parameter (C) and oscillator amplitude (A) of the Lorentz oscillator with the reduction of the QW thickness. This reflects the fact that as the QW thickness is reduced, the area under
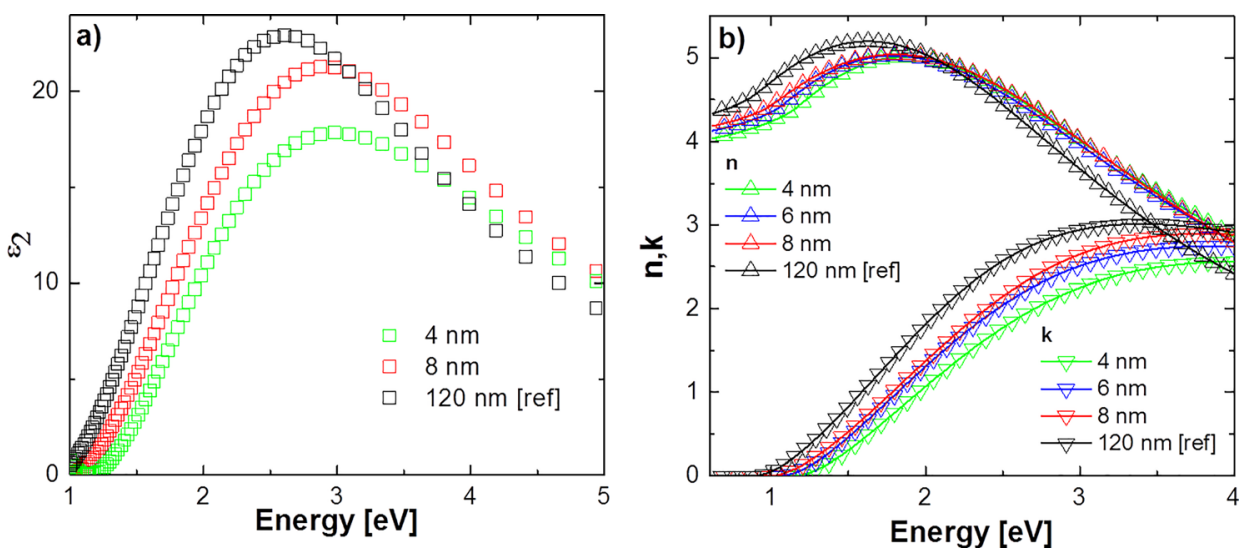

FIG. 5. $\varepsilon_{2}$ (a) and n-k spectra (b) of a-Ge films obtained by JTL model. 


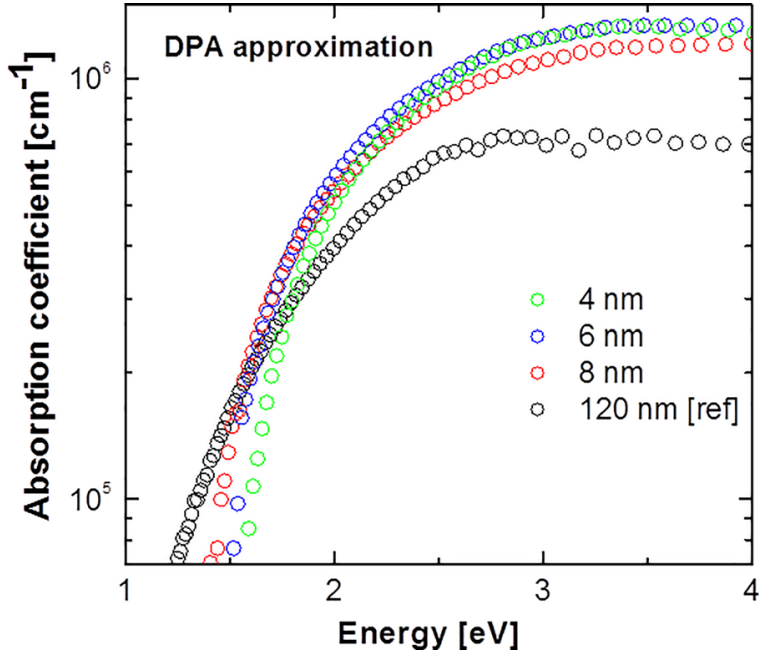

FIG. 6. The absorption coefficient of a-Ge QWs and of a reference sample extracted by DPA approach.

the Lorentz oscillator peak in the $\varepsilon_{2}$ function changes [Fig. 5(a)], as result of the modified interaction among the incoming electromagnetic field and the confined electrons, in agreement with previous observation for Ge QW. ${ }^{28}$ The broadening of the oscillator peak can also be linked to a larger roughness of the QW. Figure 5(b) reports the n-k spectra obtained within the JTL model. The QCE induced a clear blue-shift of $\mathrm{k}$ spectra, linked to the bandgap widening, and a slight modification of $n$ which indicates how the propagation of the electromagnetic field changes in confined Ge QW.

\section{B. DPA approach}

In this section, we show the results achieved by applying the DPA model. Figure 6 reports $\alpha$ spectra of a-Ge QWs and of a reference sample (120 nm thickness) as extracted by Fig. 3 after applying Eq. (6), where $\mathrm{T}^{\mathrm{sub}}$ was measured by spectrophotometry in the wavelength range from $200 \mathrm{~nm}$ to $2000 \mathrm{~nm}$. The saturation of $\alpha$ for the reference sample at $\mathrm{E}>3.4 \mathrm{eV}$ is an artifact due to the sensitivity limit of transmitted light passing through a thick absorbing layer. The most evident effect in Fig. 6 is the blue-shift occurring close to onset of absorption spectra by decreasing the QW thickness. Moreover, in the range from $1.8 \mathrm{eV}$ to $2.5 \mathrm{eV}$, the
TABLE II. The extracted bandgaps and energy ranges for the linear fit in Tauc and Cody plot applied to DPA method.

\begin{tabular}{lccccc}
\hline \hline & \multicolumn{2}{c}{ Tauc } & & \multicolumn{2}{c}{ Cody } \\
\cline { 2 - 3 } \cline { 5 - 6 } Sample $(\mathrm{nm})$ & $\mathrm{Eg}(\mathrm{eV})$ & Range fit (eV) & & Eg (eV) & Range fit (eV) \\
\hline 4 & 1.16 & $1.7-2.5$ & & 1.27 & $1.6-1.8$ \\
6 & 1.05 & $1.6-2.5$ & & 0.99 & $1.6-1.8$ \\
8 & 0.96 & $1.6-2.4$ & & 0.85 & $1.6-1.8$ \\
120 & 0.88 & $1.6-2.6$ & & 0.76 & $1-1.3$ \\
\hline \hline
\end{tabular}

magnitude of absorption coefficient in Ge QW exceeds that of $120 \mathrm{~nm}$ Ge film This result is in contrast with the decrease in $\varepsilon_{2}$ reported in Fig. 5 and it should be interpreted as an increasing error for thinner films deriving from the approximation. ${ }^{32}$ Still, this artefact does not significantly alter the determination of $E_{g}$, as shown below.

As already reported, once the absorption spectra are calculated, the optical bandgaps can be extracted by using the linear fit performed on Tauc or Cody plots (Fig. 7).

The Tauc plot shows a much wider energy range of linearity than Cody plot, probably explaining to its wider use by the scientific community. However, choosing the right range of validity is a key issue. The linear fit in the Tauc plot were performed in an energy range determined by the empirical rule for which the Tauc model can be applied for $\alpha>10^{4} \mathrm{~cm}^{-1} .17$ Given the very thin film used, the rule of $3<\alpha \mathrm{l}<10$ cannot be applied for the Cody plot. $^{21} \mathrm{We}$ performed the linear fitting for both Tauc and Cody plots, in the same energy range, for all samples. In Figs. 7(a) and 7(b), Tauc and Cody plots and their relative linear fits for all the Ge QWs (from 8 to $4 \mathrm{~nm}$ ) and reference sample are shown. Table II reports the extracted optical bandgaps and energy ranges of linear fit for Tauc and Cody plots, respectively. For Tauc plot $(0.88 \mathrm{eV}-1.16 \mathrm{eV})$ and Cody plot $(0.76 \mathrm{eV}$ $-1.27 \mathrm{eV}$ ), we observe an increase in optical bandgap as $\mathrm{Ge}$ film thickness is reduced, as expected. The Tauc plot is characterized by a unique linear region which extends over a wider spectral range with respect to the Cody plot. This allows to perform the linear fits of the Tauc plot in a much wider energy range $(1.6 \mathrm{eV}-2.5 \mathrm{eV})$ with respect to the Cody one $(1.6 \mathrm{eV}-1.8 \mathrm{eV})$.
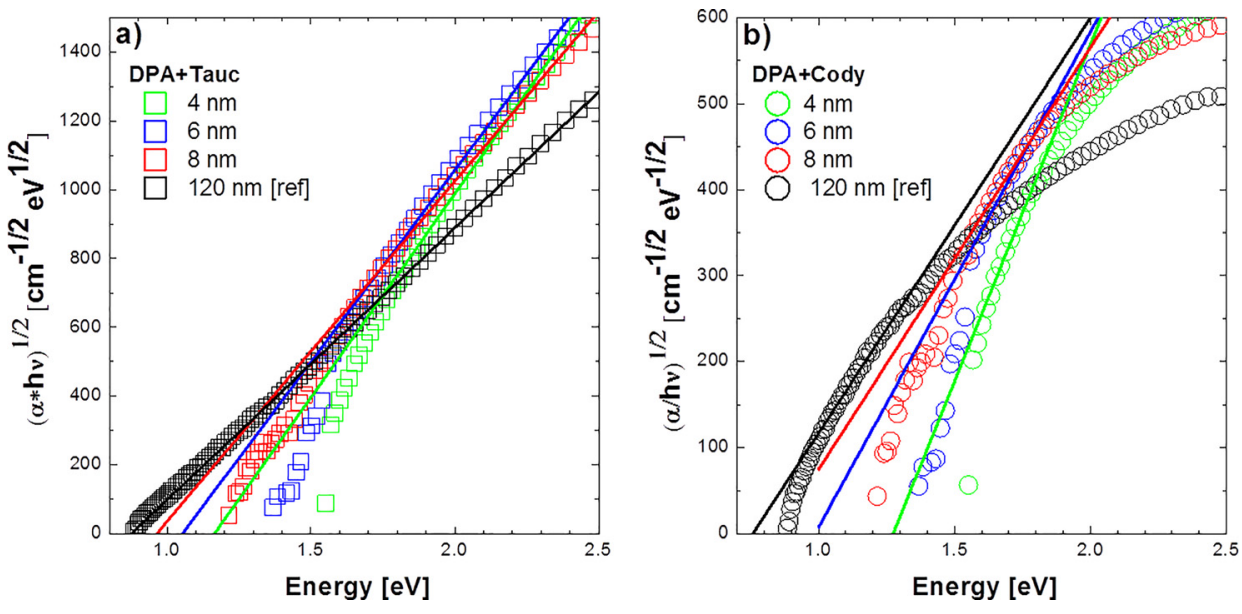

FIG. 7. Tauc (a) and Cody plots (b) and corresponding linear fit derived from data in Fig. 6. 


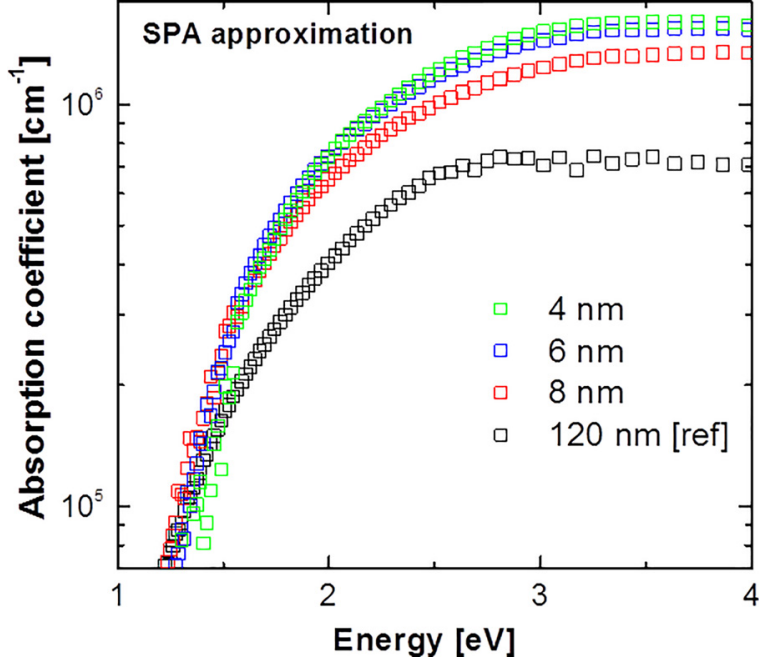

FIG. 8. The absorption coefficient of a-Ge QWs and of a reference sample extracted by SPA approach.

\section{SPA approach}

In Fig. 8, the absorption spectra of all Ge QWs and reference a-Ge bulk film, calculated by Eq. (1), are reported. As observed for DPA approach, the absorption onset shows a slight blue-shift with the reduction of the Ge film thickness.

In order to extract $\mathrm{E}_{\mathrm{g}}$, Tauc and Cody plots are used, as shown in Figs. 9(a) and 9(b). The same criteria reported in DPA section for linear fitting procedures were used here. The so-extracted values of the optical bandgap, reported in the Table III, show the expected increase with the reduction of the Ge film thickness with a larger extent for the Cody plot. As for the DPA approach, we observe that the linear region of Tauc plot is wider than for the Cody plot.

\section{RESULTS AND DISCUSSION}

In this section, the three methods are compared and discussed. In Fig. 10, we report the comparison of the absorption coefficient spectra for the $8 \mathrm{~nm}$ Ge QW, extracted with three different methods: SPA, DPA, and GTM approach. In the latter case, the $\alpha$ spectrum was calculated by using Eq. (6) with the $k$-dispersion obtained through simulation of R\&T spectra. It is clear that, in both approximated methods (SPA and DPA), the absorption coefficient is overestimated in the
TABLE III. The extracted bandgaps and energy ranges for the linear fit in Tauc and Cody plot applied to SPA method.

\begin{tabular}{lccccc}
\hline \hline \multirow{2}{*}{ Sample (nm) } & \multicolumn{2}{c}{ Tauc } & & \multicolumn{2}{c}{ Cody } \\
\cline { 2 - 3 } \cline { 5 - 6 } & Eg (eV) & Range fit $(\mathrm{eV})$ & & Eg (eV) & Range fit (eV) \\
\hline 4 & 0.91 & $1.7-2.5$ & & 1.02 & $1.3-1.75$ \\
6 & 0.87 & $1.6-2.5$ & & 0.93 & $1.3-1.7$ \\
8 & 0.84 & $1.6-2.4$ & & 0.75 & $1.2-1.7$ \\
120 & 0.86 & $1.6-2.6$ & & 0.62 & $1-1.5$ \\
\hline \hline
\end{tabular}

$1.5 \mathrm{eV}-4 \mathrm{eV}$ energy range. This overestimation of $\alpha$ is due to the fact that multiple reflections and interference effects are neglected in approximated models. This discrepancy increases in the SPA approach over the DPA one and also by reducing Ge QW thickness. In fact, for thinner films, less light is absorbed in each pass and thus multiple reflections play an increasing important role on the absorption process. ${ }^{32}$ Despite of this discrepancy, the determination of $\mathrm{E}_{\mathrm{g}}$ by DPA converges with that of the JTL model.

In Fig. 11, the $\mathrm{E}_{\mathrm{g}}$ extracted by JTL and approximated methods are compared. Figure 11(a) shows that JTL and DPA + Tauc methods give the same results, with a slight divergence appearing as the Ge QW goes below $4 \mathrm{~nm}$. Despite the significant mismatch of absorption spectra between JTL and DPA models, the optical bandgaps extracted by both methods coincides, almost perfectly. SPA + Tauc approach gives much lower $E_{g}$ values without any clear change with the thickness reduction (if $\mathrm{E}_{\mathrm{g}}$ in the reference sample is considered). DPA + Tauc approach gives more reliable data than SPA + Tauc approach. Despite of the approximation used in DPA and SPA to extract $\alpha$ from R\&T (as shown in Fig. 10), the first method, joined with Tauc plot, gives results comparable with the exact method. Figure 11(b) shows that the Cody plots vaguely catches the $\mathrm{E}_{\mathrm{g}}$ increase by reducing the QW thickness, even if a large shift of $0.2-0.3 \mathrm{eV}$ appears among the methods compared.

Applying the Cody plot to the approximated methods did not result in some convergence with the non-approximated method (GTM). In order to assess the reliability of approximated methods (SPA and DPA) and the application of the Cody model to these latter, we decided to compare the $\mathrm{E}_{\mathrm{g}}$ values extracted by approximated methods coupled with
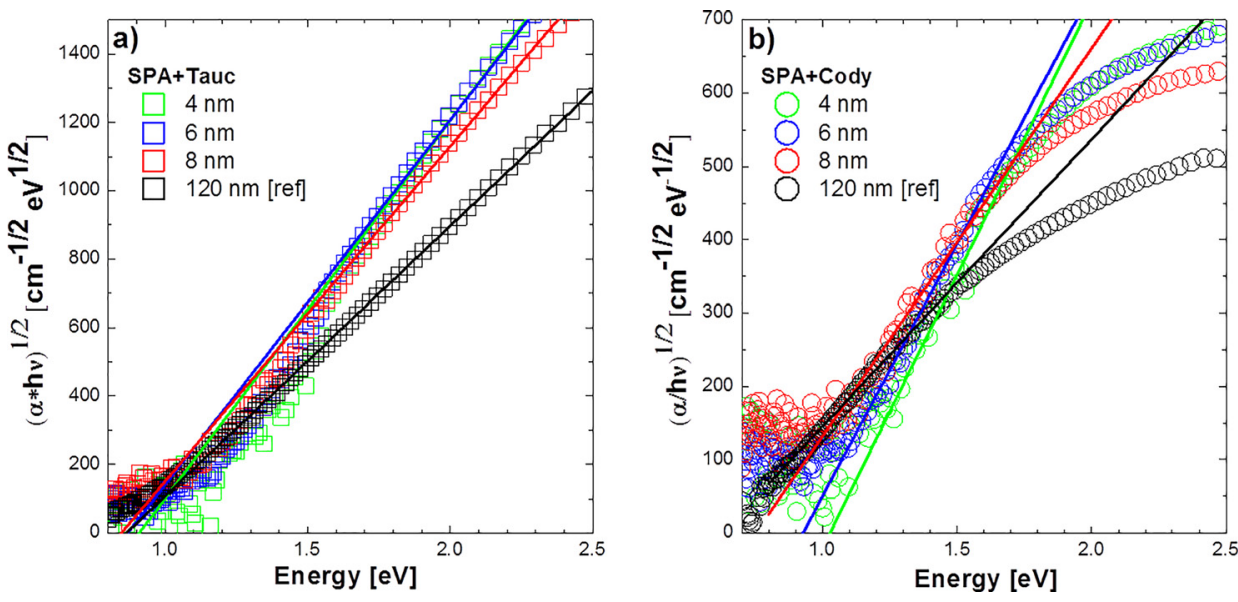

FIG. 9. Tauc (a) and Cody (b) plots and corresponding linear fit derived from data in Fig. 8. 


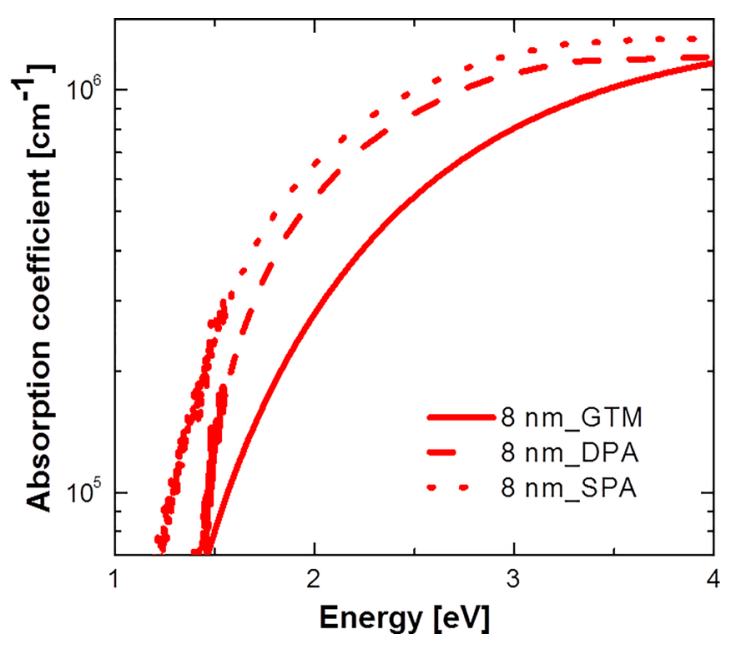

FIG. 10. The absorption coefficient spectra of $8 \mathrm{~nm}$ Ge QW extracted with three different methods: GTM (solid line), DPA (dashed line), and SPA (dotted line).

Cody plot (DPA + Cody and SPA + Cody) with the values extracted applying the Cody plot to absorption coefficient spectra extracted through GTM approach. It should be noted that when Cody plot is used, the $\mathrm{E}_{\mathrm{g}}$ values are usually lower than those with Tauc plot. Indeed, the reference sample analyzed with Cody plot gives invariably $\mathrm{E}_{\mathrm{g}}$ lower than $0.8 \mathrm{eV}$ (from $0.6 \mathrm{eV}$ to $0.7 \mathrm{eV}$ ), which does not agree with theory and experimental results on a-Ge $\left(\mathrm{E}_{\mathrm{g}}\right.$ is around $\left.0.8 \mathrm{eV}\right) .{ }^{17,33} \mathrm{It}$ should be noted that while Cody plot gave some reliable results for $\mathrm{a}-\mathrm{Si}^{18,21}$ this is not always true for other semiconductors, especially in a confined system. a-Si film is characterized by large tails in the bandgap, probably weakening the validity of Tauc assumption. Some semiconductor NSs have a high density of electronic states within the bangap, allowing a more reliable approach via the Cody plot. By studying $\mathrm{Ge}$ QWs grown by sputtering technique, Liu et al. ${ }^{19}$ reported two linear regions of the Tauc plot, each extending over a fairly narrow energy interval $(\sim 0.3$ to $0.4 \mathrm{eV})$, and a unique linear region for the Cody plots $(\sim 0.6 \mathrm{eV})$. Thus, they claimed that Cody plot gives a more unambiguous determination of $\mathrm{E}_{\mathrm{g}}$ compared to Tauc plot. ${ }^{19}$ Their conclusion is in contrast with ours, most probably because of the different growth technique used. In fact, Liu et al. prepared their Ge QWs by sputtering technique, while we used PECVD method. In a previous

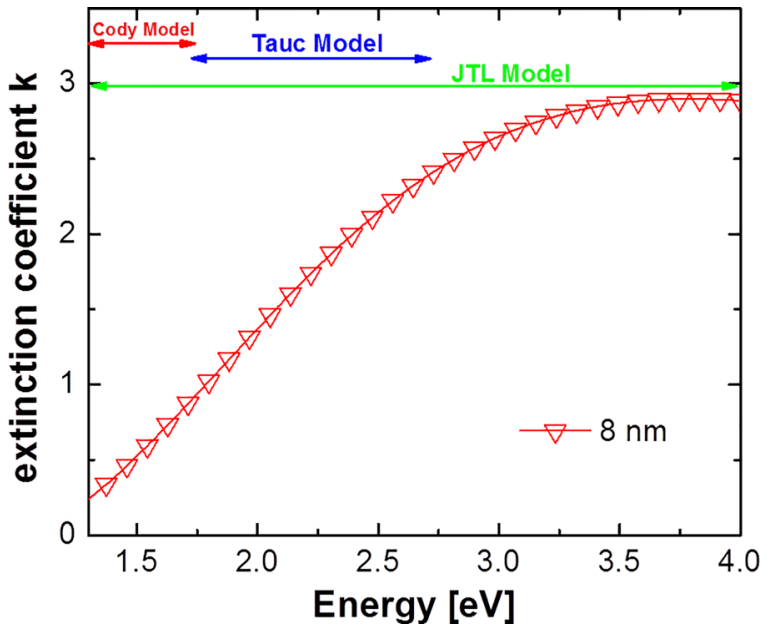

FIG. 12. Extinction coefficient $\mathrm{k}$ of $8 \mathrm{~nm}$ Ge QW with three regions of applicability of Cody (red arrow), Tauc (blue arrow), and JTL (green arrow) models.

work, we observed that PECVD QDs exhibit a sharper interface with lower amount of Ge sub-oxide states in comparison with sputter samples, ensuring a stronger electron-hole confinement into Ge QDs of PECVD samples. ${ }^{16}$ Since Tauc plot focuses the threshold energy range of absorption coefficient, which is affected by any midgap levels or bending induced by interface defects, the different behavior of our Tauc plots from those of Liu et al. can be regarded as a consequence of the different growth technique used. In our case the Tauc plot is characterized by a unique linear region which extends over a wider spectral range $(0.8 \mathrm{eV})$ with respect to the case of Ref. 19. Ge NS produced by PECVD methods were shown to be almost ideal as far as the confining potential and the $\mathrm{Ge} / \mathrm{SiO}_{2}$ interface are concerned, ${ }^{16}$ which can result in a lower density of tail states in the bandgap, accounting for the superiority of Tauc approach observed in our results. As already said earlier, care should be taken when considering the energy range for fitting. For this purpose, in Fig. 12, we report, as an example, the extinction coefficient spectrum $k$ for the $8 \mathrm{~nm} \mathrm{Ge}$ QW as obtained within the JTL model. In this graph, we identify three energy regions for the three discussed methods. The first range is for the Cody approach and goes from $1.3 \mathrm{eV}$ to $1.8 \mathrm{eV}$. For energy values greater than $1.8 \mathrm{eV}$, we have generally observed the loss of linearity in the
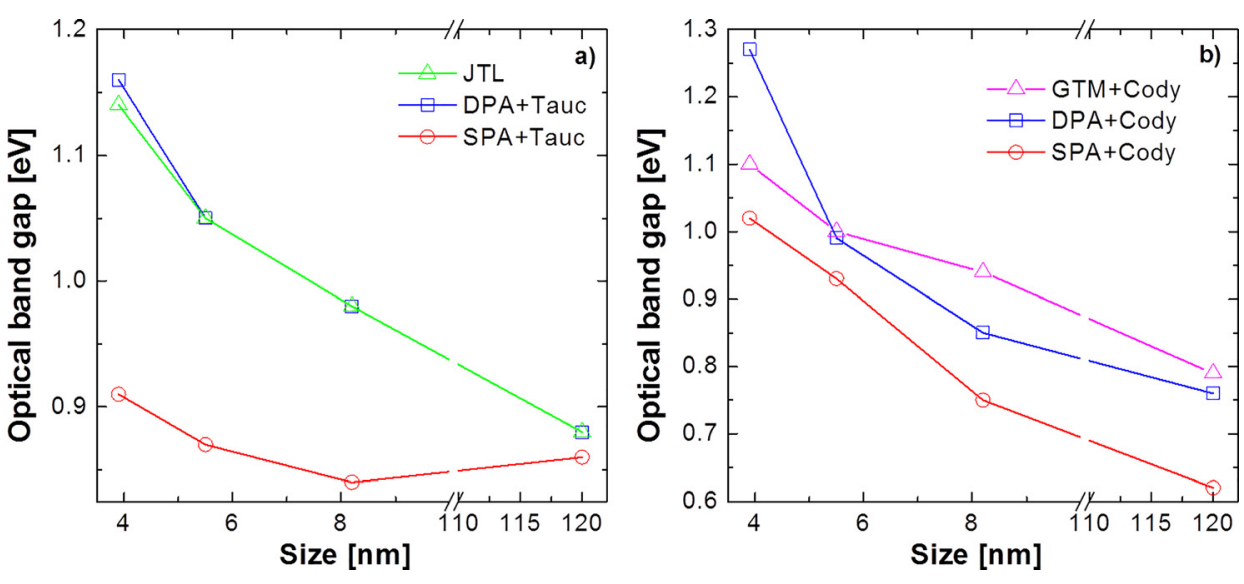

FIG. 11. (a) Comparison of optical bandgaps extracted by JTL model and by the approximated methods (DPA and SPA) by using Tauc (a) and Cody (b) plots. 


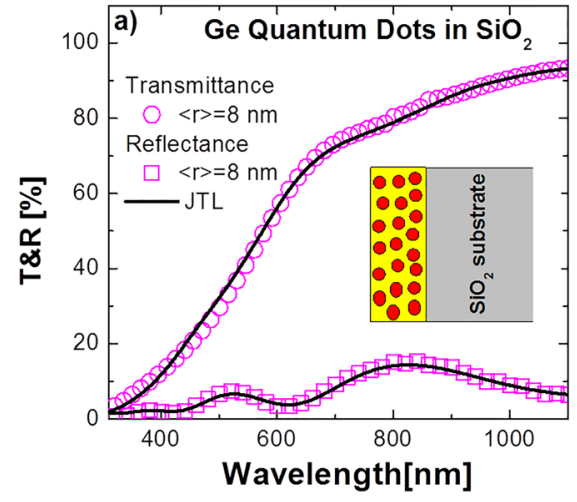

JTL parameters

\begin{tabular}{|c|c|c|c|c|c|c|}
\hline Sample & $E_{\mathrm{g}}[\mathrm{eV}]$ & $\mathrm{E}_{0}[\mathrm{eV}]$ & $\mathrm{A}$ & $\mathrm{C}[\mathrm{eV}]$ & $\mathbf{n}_{\text {inf }}$ & $\boldsymbol{\chi}^{2}$ \\
\hline$\langle\mathrm{r}>=8 \mathrm{~nm}$ & 1.29 & 2.85 & 185 & 3.8 & 1.3 & 0.81 \\
\hline
\end{tabular}

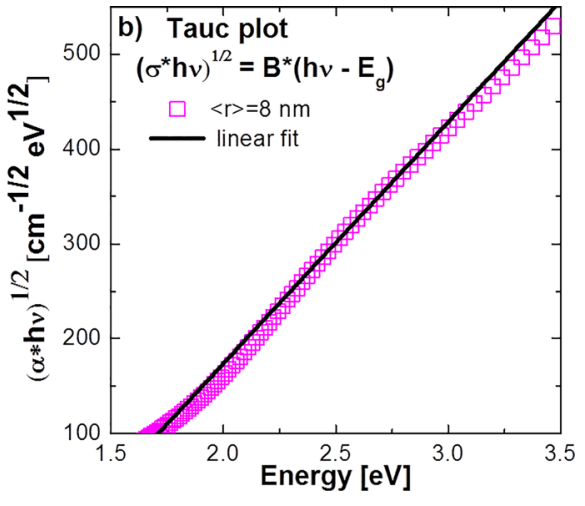

\begin{tabular}{|c|c|c|}
\hline \multirow{2}{*}{ Sample } & \multicolumn{2}{|c|}{ Tauc } \\
\cline { 2 - 3 } & Eg [eV] & Range fit \\
\hline$<\mathrm{r}>=8 \mathrm{~nm}$ & 1.32 & $2.4-2.8 \mathrm{eV}$ \\
\hline
\end{tabular}

FIG. 13. (a) Experimental (symbols) and computed (black lines) $\mathrm{T}$ and $\mathrm{R}$ spectra of an ensemble Ge QDs with mean size of $8 \mathrm{~nm}$. Table below reports the five JTL fit parameters and the $\mathrm{X}^{2}$ test: (b) Tauc plot and corresponding linear fit for $8 \mathrm{~nm} \mathrm{Ge}$ QDs. In the table the extracted bandgaps and energy ranges for the linear fit in Tauc plot are reported. The inset shows a schematic representation of the sample structure.
Cody plot. Tauc plots show a wider linear region with respect to Cody plots, ensuring a lower uncertainty in the determination of $E_{g}$ through the linear fit. Finally, the application range of JTL model spreads from $1 \mathrm{eV}$ to $4 \mathrm{eV}$, as the full R\&T spectra have been successfully fitted. The JTL approach is clearly the most powerful one, not only because it is able to fit the experimental optical data in their full energy range but also because it does not use any approximation on the extraction of $\alpha$ from R\&T data. Both Tauc and Cody plots show a limited energy range where the linear fit can be done, as they essentially focus on the light absorption close to the bandgap energy region. Still, Tauc approach is able to fit the approximated $\alpha$ over a larger range than the Cody approach, leading to smaller uncertainty in the $\mathrm{E}_{\mathrm{g}}$ determination. Finally, Tauc model applied to $\alpha$ extracted through the DPA exhibited the best performance when compared to the JTL non approximated method. This is due to the larger range of linear fit of Tauc over Cody plot and the lower degree of approximation of DPA method in extraction $\alpha$ from experimental data.

In order to check the validity of the above results in NS other than QW, we employed a sample produced by PECVD containing $\mathrm{Ge} \mathrm{QD}\left(8 \mathrm{~nm}\right.$ average diameter) in $\mathrm{SiO}_{2}$, and applied the JTL and the Tauc + DPA approach (Fig. 13). First of all, a good agreement between the experimental R\&T spectra and the simulation data was obtained in this case. The fitting JTL parameters (table in Fig. 13) are consistent with the values found above for the $8 \mathrm{~nm} \mathrm{Ge} \mathrm{QW}$ (Table I), except for the $\mathrm{E}_{\mathrm{g}}$ value which is larger for QDs, as expected given the stronger confinement (3D over $1 \mathrm{D}$ confined structure). The DPA + Tauc method gives the same value of $E_{g}$ as the JTL one, confirming that the extraction of optical band gap via the DPA + Tauc method is reliable also for $3 \mathrm{D}$ confined nanostructures.

\section{CONCLUSIONS}

Three methods to extract the optical bandgap of Ge NS from experimental R\&T spectra have been compared to evaluate their degree of accuracy and complexity. Ge QWs (4-8 nm thick) or Ge QDs (8 $\mathrm{nm}$ in diameter) embedded in $\mathrm{SiO}_{2}$ matrix were deposited by PECVD. Two methods based on double pass (DPA) or single pass (SPA) approximation are employed to extract $\alpha$ from R\&T spectra, and Tauc or Cody plots are used to evaluate $\mathrm{E}_{\mathrm{g}}$ by linear fitting. A third method, based on the Tauc Lorentz oscillator model, is used as a comparison where $\mathrm{E}_{\mathrm{g}}$ comes by a direct fitting of $\mathrm{R} \& \mathrm{~T}$ spectra by building complex refractive indexes. The DPA coupled with Tauc plot shows to be a reliable and easy method to extract $E_{\mathrm{g}}$ from $\mathrm{R} \& \mathrm{~T}$ spectra, as its results satisfactorily converge with the exact method for Ge QWs and QDs. The SPA overestimates the absorption spectra, and as a consequence, it systematically underestimates the $\mathrm{E}_{\mathrm{g}}$. On the other hand, the Tauc plot always shows a much wider range of linearity in comparison to the Cody plot, leading to a better evaluation of $E_{g}$. The superiority of the Tauc approach over the Cody one, joined with the sharp and clean $\mathrm{Ge} / \mathrm{SiO}_{2}$ interface obtained with the PECVD technique, leads to the conclusion that the constant matrix approximation used in the Tauc model well describes the light absorption process also in confined nanostructures. The reported methods have largely been used in the literature to evaluate $\mathrm{E}_{\mathrm{g}}$ in semiconductor NS, and our comparison between Ge QWs and QDs shows limits and benefits of each method.

\section{ACKNOWLEDGMENTS}

This work has been sponsored by bilateral CNRTUBITAK project "Application of nanoporous $\mathrm{Si}$ and $\mathrm{Ge}$ nanostructures to advanced solar cells" (Grant No. 211T142) and in the framework of the project ENERGETIC PON00355_3391233. Part of this work was performed at BeyondNano CNR-IMM, Italy, supported by MIUR under the project Beyond-Nano (PON a3_00363). The authors thank C. Percolla, S. Tatì, and G. Panté (MATIS CNR-IMM) for expert technical assistance.

${ }^{1}$ F. Priolo, T. Gregorkiewicz, M. Galli, and T. F. Krauss, Nat. Nanotechnol. 9, 19 (2014).

${ }^{2}$ Y. M. Niquet, G. Allan, C. Delerue, and M. Lannoo, Appl. Phys. Lett. 77, $1182(2000)$.

${ }^{3}$ A. D. Yoffe, Adv. Phys. 51, 799 (2002).

${ }^{4}$ N. Usami, W. Pan, T. Tayagaki, S. Chu, J. Li, T. Feng, Y. Hoshi, and T. Kiguchi, Nanotechnology 23, 185401 (2012). 
${ }^{5}$ S. Cosentino, E. G. Barbagiovanni, I. Crupi, M. Miritello, G. Nicotra, C. Spinella, D. Pacifici, S. Mirabella, and A. Terrasi, Sol. Energy Mater. Sol. Cells 135, 22 (2015).

${ }^{6}$ X. Liu, X. Ji, M. Liu, N. Liu, Z. Tao, Q. Dai, L. Wei, C. Li, X. Zhang, and B. Wang, ACS Appl. Mater. Interfaces 7, 2452 (2015).

${ }^{7}$ C. Y. Chien, W. T. Lai, Y. J. Chang, C. C. Wang, M. H. Kuo, and P. W. Li, Nanoscale 6, 5303 (2014).

${ }^{8}$ J. Liu, M. Beals, A. Pomerene, S. Bernardis, R. Sun, J. Cheng, L. C. Kimerling, and J. Michel, Nat. Photonics 2, 433 (2008).

${ }^{9}$ Y. Kuo, Y. K. Lee, Y. Ge, S. Ren, J. E. Roth, T. I. Kamins, D. A. B. Miller, and J. S. Harris, Nature 437, 1334 (2005).

${ }^{10}$ M.-F. Ng and R. Q. Zhang, J. Phys. Chem. B 110, 21528 (2006).

${ }^{11}$ S. Mirabella, R. Agosta, G. Franzò, I. Crupi, M. Miritello, R. Lo Savio, M. A. Di Stefano, S. Di Marco, F. Simone, and A. Terrasi, J. Appl. Phys. 106, 103505 (2009).

${ }^{12}$ R. Guerra, M. Marsili, O. Pulci, and S. Ossicini, Phys. Rev. B 84, 075342 (2011).

${ }^{13}$ S. Mirabella, S. Cosentino, A. Gentile, G. Nicotra, N. Piluso, L. V. Mercaldo, F. Simone, C. Spinella, and A. Terrasi, Appl. Phys. Lett. 101, 011911 (2012).

${ }^{14}$ P. B. Sorokin, P. V. Avramov, L. A. Chernozatonskii, D. G. Fedorov, and S. G. Ovchinnikov, J. Phys. Chem. A 112, 9955 (2008).

${ }^{15}$ G. Franzò, M. Miritello, S. Boninelli, R. Lo Savio, M. G. Grimaldi, F. Priolo, F. Iacona, C. Spinella, and S. Coffa, J. Appl. Phys. 104, 094306 (2008).

${ }^{16}$ S. Cosentino, A. M. Mio, E. G. Barbagiovanni, R. Raciti, R. Bahariqushchi, M. Miritello, G. Nicotra, A. Aydinli, C. Spinella, A. Terrasi, and S. Mirabella, Nanoscale 7, 11401 (2015)

${ }^{17}$ J. Tauc, T. Grigorovivi, and A. Vancu, Phys. Status Solidi 15, 627 (1966).

${ }^{18}$ G. D. Cody, B. G. Brooks, and B. Abeles, Sol. Energy Mater. 8, 231 (1982).
${ }^{19}$ P. Liu, P. Longo, A. Zaslavsky, and D. Pacifici, J. Appl. Phys. 119, 014304 (2016).

${ }^{20}$ S. Knief and W. von Niessen, Phys. Rev. B 59, 12940-12946 (1999).

${ }^{21}$ T. M. Mok and S. K. O'Leary, J. Appl. Phys. 102, 113525 (2007).

${ }^{22}$ M. Mayer, SIMNRA User's Guide, Report IPP 9/113 Garchin (Max Planck Institut für Plasmaphysik, 1997).

${ }^{23}$ S. Cosentino, E. Sungur Ozen, R. Raciti, A. M. Mio, G. Nicotra, F. Simone, R. Turan, A. Terrasi, A. Aydinli, and S. Mirabella, J. Appl. Phys 115, 043103 (2014).

${ }^{24}$ E. Centurioni, Appl. Opt. 44, 7532 (2005). The computer programme Optical can be downloaded at http://www.bo.imm.cnr.it/users/centurioni/ optical.html.

${ }^{25}$ G. E. Jellison, Jr. and F. A. Modine, Erratum Appl. Phys. Lett. 69, 371 (1996); 69, 2137 (1996).

${ }^{26}$ A. Allegrezza, F. Gaspari, M. Canino, M. Bellettato, A. Desalvo, and C. Summonte, Thin Solid Films 556, 105 (2014); 564, 426 (2014).

${ }^{27}$ D. V. Likhachev, N. Malkova, and L. Poslavsky, Thin Solid Films 589, 844 (2015).

${ }^{28}$ S. Cosentino, M. Miritello, I. Crupi, G. Nicotra, F. Simone, C. Spinella, A. Terrasi, and S. Mirabella, Nanoscale Res. Lett. 8, 128 (2013).

${ }^{29}$ E. D. Palik and G. Ghosh, Handbook of Optical Constants of Solids (Academic Press, San Diego, 1998).

${ }^{30}$ M. Palummo, G. Onida, and R. Del Sole, Phys. Status Solidi A 175, 23 (1999).

${ }^{31}$ S. Lee, S. Huang, G. Conibeer, and M. Green, Appl. Surf. Sci. 290, 167 (2014).

${ }^{32}$ M. Schnabel, C. Summonte, S. A. Dyakov, M. Canino, L. López-Conesa, P. Löper, S. Janz, and P. R. Wilshaw, J. Appl. Phys. 117, 045307 (2015).

${ }^{33}$ L. J. Pilione, K. Vedam, J. E. Yehoda, R. Messier, and P. J. McMarr, Phys. Rev. B 35, 9368 (1987). 